TU-410

IFP-436-UNC

August, 1992

\title{
Phenomenological Aspects of Supersymmetric Standard Models without Grand Unification
}

\author{
Satoshi Mizuta ${ }^{a}$, Daniel $\mathrm{Ng}^{(b)}$ ( and Masahiro Yamaguchi ${ }^{c}$ \\ a) Department of Physics, Tohoku University, Sendai 980, Japan \\ b) Institute of Field Physics, Department of Physics and Astronomy, \\ University of North Carolina, Chapel Hill, NC 27599-3255, USA \\ c) Department of Physics, College of General Education \\ Tohoku University, Sendai 980, Japan
}

\begin{abstract}
When the GUT relation on gaugino masses is relaxed, the mass and composition of the lightest neutralino are different from those in the GUT case. We discuss its phenomenological implications on the relic abundance of the neutralinos and on superparticle searches. In particular, we focus on the case where the neutral component of Winos dominates the lightest neutralino. It turns out the Wino-LSP is not a candidate for the dark matter.
\end{abstract}

\footnotetext{
${ }^{1}$ Address after September 1, 1992: TRIUMF, 4004 Wesbrook Mall, Vancouver, B.C., V6T 2A3, Canada
} 
In supersymmetric (SUSY) SU(5) grand-unified theories [1] and many other SUSY GUTs, a one-loop renormalization group (RG) [2] analysis gives us the following relation for the gaugino mass parameters

$$
M_{1}: M_{2}: M_{3}=5 / 3 g_{1}^{2}: g_{2}^{2}: g_{3}^{2} \simeq 1: 2: 7,
$$

where $g_{i}(i=1,2,3)$ are the gauge coupling constants of the gauge groups $U(1)_{Y}, S U(2)_{L}$ and $S U(3)_{C}$, respectively, and $M_{i}(i=1,2,3)$ the corresponding gaugino mass parameters. Note that this relation holds, independent of the particle content between the electroweak scale and the GUT scale. Therefore, eq. (11) provides an important test of the idea of the GUTs.

There are, however, many well-motivated models in which the relation (1) is not satisfied. Examples include $S U(3)_{C} \times S U(2)_{L} \times U(1)_{Y}$ string models [3] and a flipped $S U(5)$ model [4] whose gauge structure is $S U(5) \times$ $U(1)_{X}$. In the former, $M_{1}, M_{2}$ and $M_{3}$ are unknown parameters which will be determined by string dynamics, whereas in the latter, $M_{1}$ is independent of the others. Indeed in the flipped $S U(5)$ it has been argued [5] that, using a RG analysis, $\left|M_{1}\right| \gg\left|M_{2}\right|$ is favored to achieve the correct gauge symmetry breaking $S U(5) \times U(1)_{X} \rightarrow S U(3)_{C} \times S U(2)_{L} \times U(1)_{Y} \rightarrow S U(3)_{C} \times U(1)_{e m}$.

In this paper, we will consider phenomenological aspects of SUSY standard models without the GUT relation (1) which is assumed in most of the literature (see however refs. [6, 0, 8]). In particular, we are concerned with the mass ratio $r \equiv M_{1} / M_{2}$, since it is an important parameter in the neutralino mass matrix as we will explain later. We assume, as usual, that the lightest neutralino is also the lightest superparticle (LSP). To clarify its properties is thus important in SUSY phenomenology from the viewpoints of both accelerator physics and cosmology, especially when the LSP is stable due to a $Z_{2}$ symmetry called $R$-parity. In this paper we will mainly discuss the case 
of $|r|>1$, in which the neutral component of Winos can be a dominant composition of the LSP (see below). Note that this Wino-dominant LSP is never realized under the GUT assumption. On the other hand, some of the details on the case of $|r| \ll 1$, where the LSP is mostly the Bino, have been discussed in [7].

Throughout this paper, we consider the case of the minimal particle content in the SUSY standard model. With the $R$-parity conservation, the LSP, being a linear combination of four neutralinos (i.e. a Bino $\tilde{B}$, a neutral Wino $\tilde{W}_{3}$ and two neutral Higgsinos $\left.\tilde{H}_{1}, \tilde{H}_{2}\right)$ is stable and can be a candidate for the dark matter [9].

In the following, we will first investigate the mass and composition of the lightest neutralino. We will find that it is very degenerate with the lightest chargino at the tree-level for $|r|>1$, in particular when the lightest neutralino is dominated by the neutral Wino. We will examine if the neutralino is indeed lighter than the chargino after including radiative corrections. We will then discuss a cosmological implication. It will turn out when $|r|>1$ the relic abundance of the neutralino LSPs is small in most of the parameter space and the LSP is not a candidate for the dark matter of the Universe. Finally we will discuss superparticle searches in the non-GUT case.

The mass matrix of the neutralinos is parameterized by the gaugino mass parameters $M_{2}, M_{1}\left(=r M_{2}\right)$, a Higgsino mass parameter $\mu$ and the ratio of the vacuum expectation values of the two neutral Higgs bosons $\tan \beta=$ 
$v_{2} / v_{1}$ [10]. It is explicitly given by

$$
\left(\begin{array}{cccc}
M_{1} & 0 & -m_{Z} \sin \theta_{W} \cos \beta & m_{Z} \sin \theta_{W} \sin \beta \\
0 & M_{2} & m_{Z} \cos \theta_{W} \cos \beta & -m_{Z} \cos \theta_{W} \sin \beta \\
-m_{Z} \sin \theta_{W} \cos \beta & m_{Z} \cos \theta_{W} \cos \beta & 0 & -\mu \\
m_{Z} \sin \theta_{W} \sin \beta & -m_{Z} \cos \theta_{W} \sin \beta & -\mu & 0
\end{array}\right),
$$

in the $\left(\tilde{B}, \tilde{W}_{3}, \tilde{H}_{1}, \tilde{H}_{2}\right)^{T}$ basis. Here $M_{1}, M_{2}$ and $\mu$ are assumed to be real. As a convention, we take $M_{2}>0$ and $\mu$ both positive and negative. It is straightforward to diagonalize the matrix and we will denote the masses (which are taken to be positive) by $m_{\chi_{i}^{0}}(i=1, \cdots, 4)$ with $m_{\chi_{1}^{0}}<\cdots<m_{\chi_{4}^{0}}$. The lightest neutralino $\chi_{1}^{0}$ is written

$$
\begin{aligned}
& \chi_{1}^{0}=Z_{11} \tilde{B}+Z_{12} \tilde{W}_{3}+Z_{13} \tilde{H}_{1}+Z_{14} \tilde{H}_{2}, \\
& \sum_{i=1}^{4} Z_{1 i}^{2}=1
\end{aligned}
$$

where $Z_{1 i}$ are real numbers. It is convenient to define the purity of Bino and Wino component,

$$
p(\tilde{B})=Z_{11}^{2}
$$

and

$$
p(\tilde{W})=Z_{12}^{2}
$$

respectively. In fig. 1 , we plot in the $\left(M_{2}, \mu\right)$ plane the mass of the lightest neutralino, $m_{\chi_{1}^{0}}$, for (a) $r=0.1$ (b) $r=0.5$ (the GUT case) (c) $r=5$. Here we take $\tan \beta=2$. To demonstrate the composition of $\chi_{1}^{0}$, we also plot in the same figures the Bino purity $p(\tilde{B})$ for the cases (a), (b) and the Wino purity $p(\tilde{W})$ for the case (c). We find as expected that for small $r$, a gaugino-like LSP is indeed Bino-dominant, whereas for $|r|>1$, the lightest neutralino can be dominated by the neutral Wino when $M_{2} \ll|\mu|$. A neutralino with a smaller mass is realized for a smaller value of $|r|$ with $M_{2}$ and $\mu$ fixed. We 
will study the mass difference between $\chi_{1}^{0}$ and the lightest chargino $\chi_{1}^{ \pm}$later. In fig. 1, we have included the constraints from the LEP experiments [1]:

(i) for the lightest chargino mass, $m_{\chi_{1}^{ \pm}} \gtrsim m_{Z} / 2$,

(ii) $\Gamma\left(Z \rightarrow \chi_{1}^{0} \chi_{1}^{0}\right)<0.016 \mathrm{GeV}$,

(iii) $\operatorname{Br}\left(Z \rightarrow \chi_{i}^{0} \chi_{j}^{0}\right) \lesssim 1 \times 10^{-4}$ except for $(i, j)=(1,1)$.

The region below the thick line in the $\left(M_{2}, \mu\right)$ plane, which is excluded by the LEP experiments, does not depend so much on $r$ except for the region of $M_{2}<50(\mathrm{GeV})$ and $\mu<0$. If the mass difference between $\chi_{1}^{ \pm}$and $\chi_{1}^{0}$ is very small, the electron (or positron) emitted when the chargino decays to the LSP is too soft to be identified, and hence it will escape from the direct chargino search. However, such an event should be counted as an invisible Z decay. Therefore the bound $m_{\chi_{1}^{ \pm}} \gtrsim m_{Z} / 2$ is still valid in this case.

When the Wino is the dominant component of the lightest neutralino, which is realized for $|r|>1$ and $M_{2} \ll|\mu|$, we expect that the lightest neutralino is highly degenerate in mass with the lightest chargino. Indeed we can evaluate their masses when $m_{W} \ll M_{2} \ll|\mu|$ and find that they are degenerate up to the order of $m_{W}^{3}$ :

$$
M_{2}+\frac{m_{W}^{2}}{M_{2}^{2}-\mu^{2}}\left(M_{2}+\mu \sin 2 \beta\right)+\text { higher. }
$$

We also expect that the masses are quite degenerate when the neutralino is Higgsino-dominant $\left(M_{2} \gg|\mu|\right)$. In fig. 2, we show the mass difference between the lightest neutralino and the lightest chargino, $\Delta m^{(0)}=m_{\chi_{1}^{ \pm}}-$ $m_{\chi_{1}^{0}}$, at the tree-level for (a) $r=5$ and (b) $r=-5$. We see that $\chi_{1}^{0}$ and $\chi_{1}^{ \pm}$are quite degenerate in mass, namely $\left|\Delta m^{(0)}\right|<O(1)(\mathrm{GeV})$ in a large portion of the parameter space. For $r=5$, negative $\Delta m^{(0)}$ lies in the region excluded by the LEP constraints. Therefore, $\chi_{1}^{ \pm}$is always heavier than $\chi_{1}^{0}$. On the other hand, for $r=-5, \Delta m^{(0)}$ is negative in some part of the parameter 
space. However, the mass difference is less than a few tenth $\mathrm{GeV}$. We have checked that, for other choices of $r(|r|>1)$, the mass degeneracy generally occurs in the Wino- and Higgsino-dominant regions.

Because the masses are quite degenerate at the tree-level when $|r|>1$, it is important to consider radiative corrections to the mass difference to examine if the LSP is really neutral after including them. The calculation of them in a general parameter region is rather involved. Here we will give estimates for two limiting cases, i.e. $M_{2} \ll|\mu|$ and $M_{2} \gg|\mu|$. Let us first define $\Delta m=\Delta m^{(0)}+\Delta m^{(1)}$, where $\Delta m^{(1)}$ is the one-loop correction to the tree-level mass difference $\Delta m^{(0)}$. For the Wino-LSP case where $M_{2} \ll|\mu|$, the main contribution comes from gauge boson loops. $\Delta m^{(1)}$ is calculated to be

$$
\begin{aligned}
\Delta m^{(1)}= & \frac{2 g^{2} M_{2}}{(4 \pi)^{2}} \int_{0}^{1} d x(x+1)\left\{\cos ^{2} \theta_{W} \ln \frac{x^{2} M_{2}^{2}+(1-x) m_{W}^{2}}{x^{2} M_{2}^{2}+(1-x) m_{Z}^{2}}\right. \\
& \left.+\sin ^{2} \theta_{W} \ln \frac{x^{2} M_{2}^{2}+(1-x) m_{W}^{2}}{x^{2} M_{2}^{2}}\right\} .
\end{aligned}
$$

From eq. (7), we find that $\Delta m^{(1)}$ varies from $0.14(\mathrm{GeV})$ to $0.17(\mathrm{GeV})$ if we vary $M_{2}=50(\mathrm{GeV})$ to infinity.] On the other hand, when $M_{2} \gg|\mu|$, i.e. the LSP is almost a Higgsino, the gauge boson contribution to $\Delta m^{(1)}$ is

$$
\Delta m^{(1)}=\frac{\alpha}{2 \pi}|\mu| \int_{0}^{1} d x(x+1) \ln \frac{x^{2} \mu^{2}+(1-x) m_{Z}^{2}}{x^{2} \mu^{2}}
$$

which ranges from $0.21(\mathrm{GeV})$ to $0.35(\mathrm{GeV})$ if we take $|\mu|$ from $50(\mathrm{GeV})$ to infinity. From eqs. (7) and (8), we can conclude that, in large parameter regions (at least in the Wino- and Higgsino-dominant regions), the lightest neutralino is indeed lighter than the lightest chargino. The calculation in the mixed region will be complicated, which we will not discuss in this paper.

\footnotetext{
${ }^{2}$ For $M_{2} \gg m_{W}$, eq. (17) reduces to $\Delta m^{(1)}=\alpha m_{W} / 2\left(1+\cos \theta_{W}\right)+\mathcal{O}\left(m_{W}^{2} / M_{2}\right)$.
} 
There is another source which potentially gives a large radiative correction to a neutralino mass in the Higgsino LSP region. Namely, when the mass-squared mixing term $m_{\tilde{t}_{L R}}^{2}$ between the right and left stops is large, a top-stop loop will give a radiative correction to the $\tilde{H}_{2} \tilde{H}_{2}$ entry of the mass matrix

$$
\begin{aligned}
\Delta & \sim \frac{3 h_{t}^{2}}{(4 \pi)^{2}} m_{t} m_{\tilde{t}_{L R}}^{2} m_{S}^{-2} \\
& =0.66(\mathrm{GeV}) \times\left(\frac{m_{t}}{150(\mathrm{GeV})}\right)^{4}\left(\frac{m_{S}}{500(\mathrm{GeV})}\right)^{-2} \frac{A}{500(\mathrm{GeV})} \frac{1}{\sin ^{2} \beta},
\end{aligned}
$$

where $m_{t}$ is the top mass, $m_{S}$ stands for the stop mass scale and $m_{\tilde{t}_{L R}}^{2}=m_{t} A$. This can have both signs and the magnitude can be as large as $1 \mathrm{GeV}$ or more.

Let us now discuss the effects of relaxing the GUT relation on the possibility of the LSP dark matter. As we stated above, the LSP is a candidate for the dark matter. It is then an important task to calculate the cosmic relic density of the LSPs [12, 13, 14]. The case of $|r| \ll 1$ has been discussed in detail by Griest and Roszkowski [7]. They have shown that a light Bino-dominant LSP with mass $m_{\chi_{1}^{0}} \lesssim 10(\mathrm{GeV})$ survives the LEP and CDF constraints and that such a light LSP can indeed be a candidate for the dark matter of the Universe.

Here we discuss the case where $|r|$ exceeds unity and therefore the LSP can be dominated by the neutral component of the Winos, $\tilde{W}_{3}$. If this Wino-dominant LSP is lighter than the W-boson, the annihilation modes of the neutral Wino pair are quite similar to those of the Bino pair and hence one might expect that the light Wino is a candidate for the dark matter of the Universe. However this is not the case. We have seen that the neutral Wino is highly degenerate in mass with its charged counterparts, $\tilde{W}$. At 
the freeze-out temperature $T \sim m_{\chi_{1}^{0}} / 20$, the charged Winos are as rich as the neutral ones. Then we have to take account of coannihilation processes [15, 16] involving superparticles other than the LSPs, the charged Winos in our case. Since $\tilde{W}_{3}$ and $\tilde{W}$ can annihilate to a fermion pair through the coupling of $\tilde{W}_{3} \gamma^{\mu} \tilde{W} W_{\mu}^{-}$, the relic abundance is greatly reduced. We use the method of ref. 15 to calculate the relic abundance of the neutralino LSPs, taking the coannihilations into account.

In fig. 3 , we show $\Omega_{\chi} h^{2}$ where $\Omega_{\chi}$ is the ratio of the mass density of the LSPs to the critical one to close the Universe and $h(0.4 \leq h \leq 1)$ is the Hubble constant in units of $100 \mathrm{~km} / \mathrm{s} / \mathrm{Mpc}$. Here we only consider the case where the LSP is lighter than the $\mathrm{W}$ boson. We have taken $r=5$ and $\tan \beta=2$. The masses of squarks and sleptons are assumed to be $1 \mathrm{TeV}$. In our numerical calculation, we have included only quarks and leptons as the final state. We have used the tree-level mass matrices to obtain the masses of the neutralinos and charginos. The radiative correction to the mass difference $\sim 0.2(\mathrm{GeV})$ does not change our numerical results of the relic abundance. When $M_{2} \ll|\mu|$ where the LSP is the Wino, the coannihilations between the neutral and charged Winos explained above make the relic abundance of the LSPs very small. When $M_{2} \gg|\mu|$ where the LSP is almost a pure Higgsino, the relic abundance is small because of the coannihilations among the Higgsinos [16], which is quite similar to the GUT case. When the LSP is a general mixture of the four neutralinos $\left(M_{2} \approx|\mu|\right)$, the relic abundance is, in general, small because several annihilation modes of the LSP pair contribute to reduce it. Actually we can see in fig. 3 the relic abundance is too small for the LSPs to constitute the dominant component of the energy density of the Universe in the whole region of the parameter space where the LSP is 
lighter than the W-boson. So far we have fixed $\tan \beta=2$ in our calculation. However we have checked that the results are not sensitive on the choices of $\tan \beta$. We have also calculated $\Omega_{\chi} h^{2}$ for $r=2$ and $r=1.2$. In both cases, there exists a tiny region where the LSP is photino-like. It is interesting to note that this region is within the reach of LEP 200. In this tiny region, the abundance of the LSPs is sensitive on the sfermion masses $m_{\tilde{f}}$. Indeed if we take $m_{\tilde{f}}=1(\mathrm{TeV})$ the relic density exceeds the critical one, while for $m_{\tilde{f}}=100(\mathrm{GeV})$ it is very small. Except for this region, we have seen that the relic density does not depend on the sfermion masses and it is always much less than the critical density. If the LSP is more massive than the W-boson, the LSP pair annihilates to the $\mathrm{W}$-pair and the relic abundance is very small. This annihilation to the W-pair occurs even when the LSP is gaugino-like, the neutral Wino in this case. This is different from the GUT case: for a Bino-dominant LSP, this annihilation process is not effective. Thus, we can conclude when $|r|>1$ the neutralino LSP is not cosmologically interesting in most of the parameter region as far as its mass is below the $\mathrm{TeV}$ scale.[]

The relaxation of the GUT assumption (1) also affects the mass spectrum of sfermions, i.e. squarks and sleptons. In the $N=1$ supergravity scenario, it is natural to assume that the squarks and the sleptons are given a universal mass at some energy scale close to the Planck one. The actual masses get renormalized when the energy goes down to the electroweak scale. Since this RG flow [2] depends on the gaugino masses, a different mass spectrum will be obtained from that of the GUT case if we relax the gaugino

\footnotetext{
${ }^{3}$ When the mass of the LSP increases above the TeV region, $\Omega_{\chi} h^{2}$ becomes again of order unity, because there the annihilation cross section is proportional to $m_{\chi_{1}^{0}}^{-2}$ and as a result $\Omega_{\chi} h^{2} \propto m_{\chi_{1}^{0}}^{2}$. But such a heavy LSP is not interesting if we recall that the low-energy SUSY provides a solution of the naturalness problem.
} 
mass relation imposed by GUT.

In fig. 1, we have shown that the LSP composition depends strongly on $r$. As a result, superparticle searches become more complicated. Here, we will consider the chargino production in electron-positron collision, $e^{+} e^{-} \rightarrow \chi_{1}^{+} \chi_{1}^{-}$ at LEP-200 where the heavier chargino $\chi_{2}^{ \pm}$will be too heavy to be accessible in most of the parameter space. Note that the chargino production does not depend on the parameter $r$. Some details on the chargino production at LEP-200 can be found in ref. [17]. To distinguish the models with different values of $r$, we have to look at the decay spectrum. The decays of charginos are numerous, we will restrict ourselves to the following signature

$$
e^{+} e^{-} \rightarrow \chi_{1}^{+} \chi_{1}^{-}, \chi_{1}^{+} \rightarrow \chi_{1}^{0} l^{+} \nu
$$

We plot the energy and angular distribution for $r=0.5$ (solid), 0.25 (dot) and 1 (dash) in fig. 4 with $\tan \beta=2, M_{2}=100(\mathrm{GeV})$ and $\mu=250(\mathrm{GeV})$. The decay of $\chi_{1}^{+}$involves the exchanges of a $\mathrm{W}$ gauge boson, a sneutrino and a selectron. The scalar lepton masses are assumed to be $100 \mathrm{GeV}$. We find that for larger $r$, the energy of the charged lepton is softer and the forward-backward asymmetry is smaller.

When $|r|$ exceeds unity, the mass of the chargino can be nearly degenerate with the LSP mass, and therefore an electron emitted when the chargino decays is too soft to be identified. This is actually a difficulty in the chargino search even in the GUT case, where the mass degeneracy occurs in the Higgsino-dominant LSP region. Note, however, in the present case that the lifetime of the chargino is roughly given by

$$
\tau=\Gamma\left(\chi_{1}^{+} \rightarrow \chi_{1}^{0} e^{+} \nu\right)^{-1} \sim 10^{-8} \sec \left(\frac{0.2 \mathrm{GeV}}{\Delta m}\right)^{5}
$$

where $\Delta m$ denotes the mass difference between the lightest chargino and the 
neutralino LSP. If the mass difference is smaller than about $200(\mathrm{MeV})$ which can be realized for $|r|>1$ as we have examined, we have a good chance to trap the chargino in a detector.

In summary, we have discussed some phenomenological aspects of the SUSY standard models when the GUT relation on the gaugino masses is relaxed. Firstly we have pointed out that the mass and composition of the lightest neutralino, which is presumably the LSP, are sensitive on the parameter $r=M_{1} / M_{2}$. In particular, when $|r|>1$ the neutral Wino can be a dominant component of the LSP. In this case, the mass of the lightest chargino is very close to that of the lightest neutralino. We have examined that in most of the parameter space the neutralino is lighter than the chargino after including the radiative corrections to the mass difference. Typically the mass difference is of order $100(\mathrm{MeV})$. We have then discussed the cosmic relic density of the neutralino LSPs. We have shown that, unlike the $|r|<1$ case, it is small in most of the parameter space when $|r|>1$ and the neutralino LSP can not be an interesting candidate for the dark matter of the Universe. In our discussion, the coannihilation processes play an essential role in reducing the relic density of the LSPs in the Wino- and Higgsinodominant LSP regions. The superparticle searches are also affected by the parameter $r$. In the chargino search, we may be able to determine $r$ by looking at the decays of the chargino. When the mass difference between $\chi_{1}^{0}$ and $\chi_{1}^{ \pm}$is smaller than $\sim 200(\mathrm{MeV})$, the lifetime of the chargino becomes long so that it will leave a spectacular track in a detector.

We would like to acknowledge T. Yanagida for reading of the manuscript and useful comments. Two of us (S.M. and M.Y.) are grateful to K. Inoue, M. Kawasaki, H. Murayama, X. Tata and T. Yanagida for helpful discussions. 
The work of D.N. was supported in part by the U.S. Department of Energy under Grant No. DE-FG05-85ER-40219. 


\section{References}

[1] S. Dimopoulos and H. Georgi, Nucl. Phys. B193 (1981) 150; N. Sakai, Z. Phys. C11 (1981) 153.

[2] K. Inoue, A. Kakuto, H. Komatsu and S. Takeshita, Prog. Theor. Phys. 68 (1982) 927.

[3] L.E. Ibáñez and D. Lüst, CERN preprint CERN-TH.6380 (1992).

[4] I. Antoniadis, J. Ellis, J.S. Hagelin and D.V. Nanopoulos, Phys. Lett. B194 (1987) 231.

[5] M. Drees, Phys. Lett. B206 (1988) 265.

[6] M. Drees and X. Tata, Phys. Rev. D43 (1991) 2971.

[7] K. Griest and L. Roszkowski, CERN preprint CERN-TH.6181/91 (September 1991).

[8] M. Yamaguchi, North Carolina preprint IFP-426-UNC (February 1992) unpublished.

[9] H.Goldberg, Phys. Rev. Lett. 50 (1983) 1419; L.M. Krauss, Nucl. Phys. B227 (1983) 556; J. Ellis, J.S. Hagelin, D.V. Nanopoulos, K.A. Olive and M. Srednicki, Nucl.Phys. B238 (1984) 453.

[10] J. Gunion and H. Haber, Nucl. Phys. B272 (1986) 1.

[11] ALEPH Collaboration, D. Decamp et al, CERN preprint CERNPHE/91-149 (1991); P. Langacker, Pennsylvania preprint UPR-0492T (January 1992).

[12] K. Griest, Phys. Rev. D38 (1988) 2357. E: D39 (1989) 3802.

[13] K. Griest, M. Kamionkowski and M.S. Turner, Phys. Rev. D41 (1990) 3565. 
[14] K.A. Olive and M. Srednicki, Phys. Lett. B230 (1989) 78; Nucl. Phys. B355 (1991) 208.

[15] K. Griest and D. Seckel, Phys. Rev. D43 (1991) 3191.

[16] S. Mizuta and M. Yamaguchi, Tohoku preprint TU-409 (July 1992).

[17] A. Bartl, H. Fraas, W. Majerotto and B. Mösslacher, Z. Phys. C55 (1992) 257. 


\section{Figure captions}

Fig. 1. Contour plots of the mass and the composition of the LSP in the $\left(M_{2}, \mu\right)$ plane when $r=$ (a) 0.1 , (b) 0.5 (the GUT case) and (c) 5. Solid lines show the same mass contours and dot lines show the purity of the Bino $p(\tilde{B})$ for $(\mathrm{a})$ and $(\mathrm{b})$ and of the Wino $p\left(\tilde{W}_{3}\right)$ for $(\mathrm{c})$ defined in the text. We have taken $\tan \beta=2$. The region below the thick line is excluded by the LEP experiments.

Fig. 2. Contour plots of the mass difference between the lightest chargino and the lightest neutralino at the tree-level, $\Delta m^{(0)}$, when (a) $r=5$ and (b) $r=-5$. We have taken $\tan \beta=2$.

Fig. 3. The relic abundance of the neutralino LSPs when $r=5$ and (a) $\mu>0$ (b) $\mu<0$. We have taken $\tan \beta=2$, the mass of the pseudoscalar Higgs boson at $1 \mathrm{TeV}$ and the mass of the sfermions at $1 \mathrm{TeV}$. The region labelled by LEP is excluded by the LEP experiments. The LSP is heavier than the $\mathrm{W}$ boson in the region labelled by " $m_{\chi}>m_{W}$ ", in which we do not calculate the relic abundance. Notice that, in the region presented here, $\Omega_{\chi} h^{2}$ is significantly small.

Fig. 4. The energy and angular distributions of the electron emitted by the leptonic decay of the chargino for $r=0.5$ (solid), 0.25 (dot) and 1 (dash). We have taken $\tan \beta=2, M_{2}=100(\mathrm{GeV})$ and $\mu=250(\mathrm{GeV})$. The scalar lepton masses are taken to be $100(\mathrm{GeV})$. 